\title{
The angular sizes of dwarf stars and subgiants (Research Note)
}

\section{Non-linear surface brightness relations in $B V R_{c} I_{c}$ from interferometry ${ }^{\star}$}

\author{
P. Kervella ${ }^{1}$ and P. Fouqué ${ }^{2}$ \\ 1 LESIA, Observatoire de Paris, CNRS UMR 8109, UPMC, Université Paris Diderot, 5 Place Jules Janssen, 92195 Meudon, France \\ e-mail: Pierre.Kervella@obspm. fr \\ ${ }^{2}$ LATT, Université de Toulouse, CNRS, 14 avenue Edouard Belin, 31400 Toulouse, France
}

Received 3 June 2008 / Accepted 9 September 2008

ABSTRACT

\begin{abstract}
Context. The prediction of stellar angular diameters from broadband photometry plays an important role in different applications. In particular, long-baseline interferometry, gravitational microlensing, extrasolar planet transits, and many other observing techniques require accurate predictions of the angular size of stars. These predictions are based on the surface brightness-color (SBC) relations. Aims. Our goal is to calibrate general-purpose SBC relations using visible colors, the most commonly available data for most stars. Methods. We compiled the existing long-baseline interferometric observations of nearby dwarf and subgiant stars and the corresponding broadband photometry in the Johnson $B V$ and Cousins $R_{\mathrm{c}} I_{\mathrm{c}}$ bands. We then adjusted polynomial SBC models to these data. Results. Due to the presence of spectral features that depend on the effective temperature, the SBC relations are usually not linear for visible colors. We present polynomial fits that can be employed with $B V R_{\mathrm{c}} I_{\mathrm{c}}$ based colors to predict the limb-darkened angular diameters (i.e. photospheric) of dwarf and subgiant stars with a typical accuracy of $5 \%$.

Conclusions. The derived polynomial relations provide a satisfactory approximation to the observed surface brightness of dwarfs and subgiants. For distant stars, the interstellar reddening should be taken into account.
\end{abstract}

Key words. stars: fundamental parameters - techniques: interferometric

\section{Introduction}

The surface brightness-color (hereafter SBC) relations link the emerging flux per solid angle unit of a light-emitting body to its color. They express the Stefan-Boltzmann relation between total brightness and effective temperature in measurable quantities such as angular diameter, magnitude and color. It assumes that temperature and bolometric correction can be translated into a color measurement. These relations have many astrophysical applications, such as the estimation of Cepheid distances (through the Baade-Wesselink method), extrasolar planet transit studies or the characterization of microlensing sources. Different colors produce tighter or more dispersed relations, because color also depends on other variables such as gravity or metallicity, at different levels depending on the adopted photometric bands. It has been shown that a combination of visible and near-IR bands, such as $(V-K)$, is probably optimal (see, e.g., Fouqué \& Gieren 1997, in the case of Cepheids). However, near-infrared photometry is not always available, for instance due to catalogue incompleteness or field crowding of IR atlases such as 2MASS in the Galactic disk. As a consequence, SBC relations in the visible domain remain very useful.

Although they are relatively similar for stars of different luminosity classes, the literature gives appropriate relations for Cepheids (Kervella et al. 2004c), giants (van Belle 1999; Nordgren et al. 2002), M giants and supergiants (van Belle et al. 1999; Groenewegen 2004), and dwarf or subgiant stars

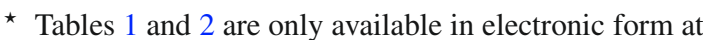
http://www. aanda.org
}

(Kervella et al. 2004b, hereafter K04). Here we limit our discussion to dwarf stars and subgiants, and revise and extend K04's work, which was based on Johnson photometric bands and was limited to linear SBC relations. In the present Note, we calibrate the SBC relations based on Cousins $R_{\mathrm{c}}$ and $I_{\mathrm{c}}$ photometric bands, in more common use than Johnson's $R$ and $I$, and we adjust non-linear SBC relations that are a better fit to the observations than linear laws. In this process, we exclusively use direct interferometric angular diameter measurements, thus avoiding cross-correlations with previously calibrated SBC relations.

\section{Interferometric and photometric data}

We collected from the literature all the available interferometric angular diameter measurements of dwarf and subgiant stars. From this list, we removed the stars for which the angular diameter was uncertain by more than 5\%. The major sets of new interferometric measurements of dwarf and subgiant stars since K04 are from Berger et al. (2006), Baines et al. (2008), Boyajian et al. (2008) and Kervella et al. (2008), all four based on measurements obtained with the CHARA array (ten Brummelaar et al. 2005). The only star from Berger et al. (2006) that has an angular diameter more precise than 5\%, GJ 15A, was removed from our list as it is a flare star, and its photometry is therefore uncertain. This was also the case for several other M dwarfs. Most of the candidate stars present variability at a certain level. In order to reject those stars for which the variability amplitude could be large, we checked their variability status in the 
GCPD (Mermilliod et al. 1997) and the GCVS (Samus 2008). All stars listed in " $\gamma$ Cas" or "UV Ceti" classes were rejected. Low amplitude " $\delta$ Scuti", such as $\alpha$ Lyr or $\beta$ Leo, "BY Dra" like $\epsilon$ Eri, GJ 570 A, GJ 699, 61 Cyg A, HR 511, or possible "RS Cvn", such as $\delta$ Eri, have been kept. Note that DY Eri (a "UV Ceti" type flare star) is not GJ $166 \mathrm{~A}$, as stated in the SIMBAD database, but GJ $166 \mathrm{C}$, therefore we kept the former in our list. We also removed the known very fast rotating stars $\left(v \sin i \gtrsim 100 \mathrm{~km} \mathrm{~s}^{-1}\right)$, including the pole-on rotator $\alpha$ Lyr (Aufdenberg et al. 2006; Peterson et al. 2006). The effective temperature of fast rotating stars is inhomogeneous over their apparent disk, they can be surrounded by circumstellar material (Kervella \& Domiciano de Souza 2006) and their photospheres can be very distorted (Monnier et al. 2007). All these phenomena combine to bias their surface brightness compared to normal stars. Overall, our selection procedure resulted in the removal of about one third of the available interferometric measurements (Table 1).

The limb darkened (LD) angular diameters were available in all cases from the original publication, except the measurementd of Sirius by Hanbury Brown et al. (1974) and Davis \& Tango (1986), for which we applied the LD correction by Claret (2000), as discussed in Sect. 5.4 of Kervella et al. (2004a). For Mozurkewich et al.'s (2003) measurements we considered the combined LD values listed by these authors. In all cases, we chose to keep the original LD values instead of correcting the uniform disk values by recomputing the limb darkening corrections, because there is a remarkable concensus in the literature to use the predictions from Kurucz's atmosphere models, approximated using Claret's laws (Claret et al. 1995; Claret 1998, 2000) to estimate the brightness distribution over the stellar disk. In fact, all the listed angular diameters were computed using these LD models, except the recent measurement of $\alpha$ Cen B published by Bigot et al. (2006) who employed 3D hydrodynamical models. Even in this case, the derived angular diameter was less than $1 \sigma$ away from the $1 \mathrm{D}$ Kurucz model result. Claret (2008) recently showed that theoretical atmosphere models do not perfectly reproduce the observed stellar intensity profiles from transiting exoplanets and eclipsing binaries. However, these discrepancies are negligible for our purpose, considering the star-to-star residual dispersion with respect to the adjusted empirical laws (Sect. 3).

The starting point for the photometry listed in Table 2 is the Hipparcos Input Catalogue (1993) as retrieved from SIMBAD, which lists $V$ and $B-V$, and the Hipparcos and Tycho Catalogues (1997), which list $V-I$. Notes in these catalogues give the origin of the listed values. When original photometry in Cousins system exists for $V-R_{\mathrm{c}}$ and $V-I_{\mathrm{c}}$, it was checked from the online version of the Lausanne General Catalogue of Photometric Data (1997), averaged over original sources according to their number of measurements, and finally combined with the $V$ band Hipparcos photometry to get $R_{\mathrm{c}}$ and $I_{\mathrm{c}}$ magnitudes. More details are given in the Notes of Table 2 .

\section{Surface brightness-color relations}

Unlike visible-infrared colors from K04, the dependence of the zero-magnitude limb-darkened angular diameter (ZMLD, defined for $m_{\lambda}=0$ ) as a function of the color is non linear in the visible. For this reason, we selected polynomial SBC relation models of the form:

$$
\log \left(\theta_{\mathrm{LD}}\right)=a_{0}+a_{1} C+a_{2} C^{2}+a_{3} C^{3}+a_{4} C^{4}-0.2 m_{\lambda}
$$
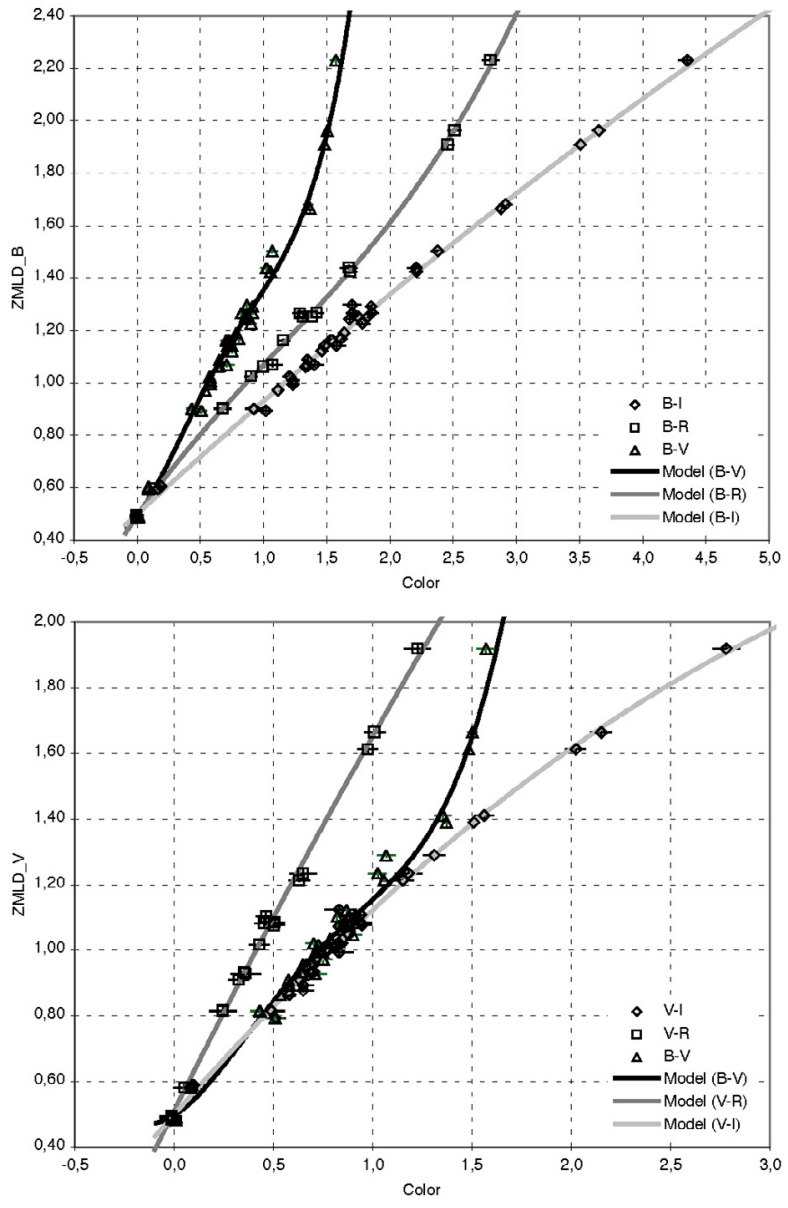

Fig. 1. Adjusted polynomial relations giving the zero-magnitude limbdarkened disk angular diameter $\left(\mathrm{ZMLD}_{\lambda}\right)$ in the $B$ and $V$ bands as a function of different color combinations. The corresponding polynomial coefficients are listed in Table 3.

where $C$ is the color of the star from two dereddened photometric bands among $B V R_{\mathrm{c}} I_{\mathrm{c}}$ (e.g. $\left.B-V, V-R_{\mathrm{c}}, \ldots\right), m_{\lambda}$ the dereddened magnitude of the star in one of the bands, and $\theta_{\mathrm{LD}}$ the limb-darkened angular diameter, measured in milliarcseconds (mas). Note that this expression is independent of the distance of the star. For the empirical fit to the observations, we selected the smallest polynomial degree that gave a satisfactory fit to all the data points. This led us to select a higher degree (four) for the $B-V$ based polynomial fits.

We would like to stress that the computed relations are valid only for a given range of colors, with only little margin for extrapolation. In particular, the SBC laws undergo steep variations for red objects. The results of the fits are presented in Fig. 1 for $B$ and $V$ and in Fig. 2 for $R_{\mathrm{c}}$ and $I_{\mathrm{c}}$. The polynomial coefficients and the domain of validity of each relation are listed in Table 3. The residual dispersions $\sigma\left(\theta_{\mathrm{LD}}\right) / \theta_{\mathrm{LD}}$ give the relative accuracy of the angular diameter predictions for each relation.

\section{Discussion}

\subsection{Reddening corrections and metallicity}

When a SBC relation is applied to a star suffering some amount of absorption, its colors must be corrected for extinction before applying the relation. The amount of extinction is generally measured by the color excess $E(B-V)$, and the conversion to other photometric bands depends on the intrinsic color of the star and 
Table 3. Polynomial coefficients of the adjusted surface brightness-color relations: $\log \left(\theta_{\mathrm{LD}}\right)=a_{0}+a_{1} C+a_{2} C^{2}+a_{3} C^{3}+a_{4} C^{4}-0.2 m_{\lambda}\left(\theta_{\mathrm{LD}}\right.$ expressed in milliarcseconds). $N$ is the number of measurements used for the calibration. The listed $C_{\min }$ and $C_{\max }$ values correspond to the minimum and maximum color index of the stars included in the polynomial fitting procedure. They represent the limits outside of which the adjusted relations are unreliable and should not be employed. The standard deviation of the residuals of the fit in log scale is listed in the $\sigma\left(\log \theta_{\mathrm{LD}}\right) \operatorname{column}$, and the corresponding relative uncertainties on the predicted angular diameter is given in the rightmost column, in percentage.

\begin{tabular}{cccrcccccccc}
\hline \hline$\lambda$ & $C$ & $N$ & $C_{\min }$ & $C_{\max }$ & $a_{0}$ & $a_{1}$ & $a_{2}$ & $a_{3}$ & $a_{4}$ & $\sigma\left(\log \theta_{\mathrm{LD}}\right)$ & $\sigma\left(\theta_{\mathrm{LD}}\right) / \theta_{\mathrm{LD}}$ \\
\hline$B$ & $B-V$ & 42 & 0.01 & 1.57 & 0.4952 & 0.5809 & 1.3259 & -1.7191 & 0.6715 & 0.0331 & $7.9 \%$ \\
$B$ & $B-R_{\mathrm{c}}$ & 21 & 0.00 & 2.80 & 0.4922 & 0.6933 & -0.1639 & 0.0486 & & 0.0172 & $4.0 \%$ \\
$B$ & $B-I_{\mathrm{c}}$ & 42 & -0.01 & 4.35 & 0.4996 & 0.4424 & -0.0115 & & & 0.0213 & $5.0 \%$ \\
$V$ & $B-V$ & 42 & 0.01 & 1.57 & 0.4952 & 0.3809 & 1.3261 & -1.7192 & 0.6716 & 0.0331 & $7.9 \%$ \\
$V$ & $V-R_{\mathrm{c}}$ & 21 & -0.01 & 1.23 & 0.5100 & 1.2159 & -0.0736 & & & 0.0191 & $4.5 \%$ \\
$V$ & $V-I_{\mathrm{c}}$ & 42 & -0.02 & 2.78 & 0.4992 & 0.6895 & -0.0657 & & & 0.0238 & $5.6 \%$ \\
$R_{\mathrm{c}}$ & $B-R_{\mathrm{c}}$ & 21 & 0.00 & 2.80 & 0.4922 & 0.4933 & -0.1639 & 0.0486 & & 0.0172 & $4.0 \%$ \\
$R_{\mathrm{c}}$ & $V-R_{\mathrm{c}}$ & 21 & -0.01 & 1.23 & 0.5100 & 1.0159 & -0.0736 & & & 0.0191 & $4.5 \%$ \\
$R_{\mathrm{c}}$ & $R_{\mathrm{c}}-I_{\mathrm{c}}$ & 21 & -0.01 & 1.55 & 0.4989 & 1.2300 & -0.3549 & & & 0.0214 & $5.1 \%$ \\
$I_{\mathrm{c}}$ & $B-I_{\mathrm{c}}$ & 42 & -0.01 & 4.35 & 0.4996 & 0.2424 & -0.0115 & & & 0.0213 & $5.0 \%$ \\
$I_{\mathrm{c}}$ & $V-I_{\mathrm{c}}$ & 42 & -0.02 & 2.78 & 0.4992 & 0.4895 & -0.0657 & & & 0.0238 & $5.6 \%$ \\
$I_{\mathrm{c}}$ & $R-I_{\mathrm{c}}$ & 21 & -0.01 & 1.55 & 0.4989 & 1.0300 & -0.3549 & 0.0291 & & 0.0214 & $5.1 \%$ \\
\hline
\end{tabular}
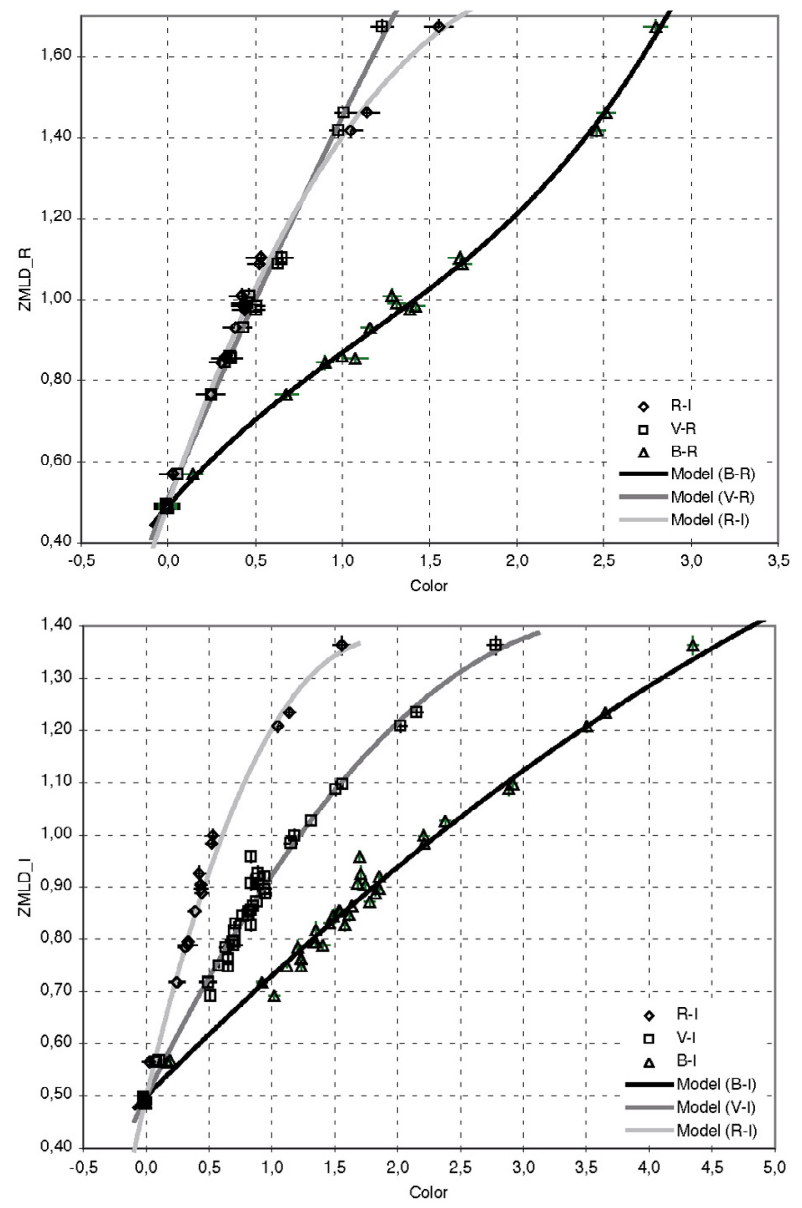

Fig. 2. Adjusted polynomial relations in the $R_{\mathrm{c}}$ and $I_{\mathrm{c}}$ bands.

on the amount of extinction (Dean et al. 1978). For blue stars, the color excess ratios are typically $E\left(V-R_{\mathrm{c}}\right)=0.60 E(B-V)$ and $E\left(V-I_{\mathrm{c}}\right)=1.25 E(B-V)$. For red stars, the coefficients may increase by up to $10 \%$.

All the stars in our list are very nearby. On average, we do not expect a significant deviation of their metallicity compared to that of the Sun. The exoplanet stars observed by Baines et al. (2008) probably present a slight overmetallicity, but the effect on the dispersion of the adjusted SBC relations is taken into account in the dispersions stated in Table 3. As discussed by $\mathrm{K} 04$, the effect of metallicity on the visible-infrared SBC relations is undetectable. For the visible SBC relations presented here, different metallicities will translate into more significant photometric color differences. However, we expect them to be small compared to the measured intrinsic dispersion of the relations, at least over a \pm 0.5 dex range around solar metallicity.

\subsection{Application to transits and microlensing}

The existence of exoplanetary transits in front of a star allows one to retrieve its linear radius, as well as the planetary radius. However, when the parallax is not known accurately and/or the star is too faint for high accuracy angular velocity measurements (from high resolution spectroscopy), they are based on an a priori radius for the star. The relations established in the present Research Note are useful in this context, as they allow one to retrieve the angular size of faint stars for which only broadband photometry is available. As a test, we can apply the $\left(V, V-I_{\mathrm{c}}\right)$ relation to the nearby transiting exoplanet stars HD 189733, for which $V=7.686 \pm 0.007$, and $\left(V-I_{\mathrm{c}}\right)=0.93 \pm 0.01$ (from Hipparcos). Due to the proximity of this star, we neglect the reddening. For these bands, the surface brightness relation is (Table 3):

$$
\log \left(\theta_{\mathrm{LD}}\right)=0.4992+0.6895\left(V-I_{\mathrm{c}}\right)-0.0657\left(V-I_{\mathrm{c}}\right)^{2}-0.2 V(2)
$$

and we obtain $\theta_{\mathrm{LD}}(\mathrm{HD} 189733)=0.352 \pm 0.020$ mas. The attached $6 \%$ uncertainty contains the photometric uncertainties and the intrinsic dispersion of the $\left(V, V-I_{\mathrm{c}}\right)$ relation. Together with the Hipparcos parallax of this star $\pi=51.94 \pm 0.87$ mas, we obtain a linear photospheric radius of $0.728 \pm 0.044 R_{\odot}$. This is in excellent agreement with the radius derived from $H S T$ transit observations by Pont et al. (2007) of $0.755 \pm 0.011 R_{\odot}$. We can also compare the $\left(V, V-I_{\mathrm{c}}\right)$ angular diameter to the one computed from the infrared $(V, V-K)$ linear relations calibrated by $\mathrm{K} 04$. The 2MASS catalogue (Skrutskie et al. 2006) lists $K=5.541 \pm$ 0.021 , giving $\theta_{\mathrm{LD}}(\mathrm{HD} 189733)=0.372 \pm 0.007$ mas. Although this value is within $1 \sigma$ of the $\left(V, V-I_{\mathrm{c}}\right)$ value, the uncertainty on this visible-infrared prediction is only one third of the visible version. A complete review of the properties of this system can be found in Torres et al. (2008).

Another important application of SBC relations is related to gravitational microlensing studies, when one needs to estimate the source radius from color-magnitude diagram (CMD) 
in the direction of the Galactic Bulge. Deredenned source magnitude and color are obtained from the relative position of the source in a CMD, with respect to the mean position of the Red Giant Clump (RGC). As the intrinsic position of the RGC is calibrated from nearby stars by Hipparcos, the assumption that the source suffers the same amount of extinction as the clump avoids the need for an estimate of the extinction (see Yoo et al. 2004, for details). Generally, the magnitudes are measured in the $I_{\mathrm{c}}$ band, and the measured color is $\left(V-I_{\mathrm{c}}\right)$, because this is what OGLE measures. A caveat of K04's SBC relations is that they are based to the Johnson $R$ and $I$ bands, and this has been a source of confusion in the past. Let's take the example of the recently discovered 3 Earth mass planet event MOA-2007-BLG192 (Bennett et al. 2008). The source appears to be fainter than the RGC by $5.7 \mathrm{mag}$ in $I_{\mathrm{c}}$, and redder by $0.07 \mathrm{mag}$ in $\left(V-I_{\mathrm{c}}\right)$. At the source distance $(7.5 \mathrm{kpc})$, the RGC is at $I_{\mathrm{c} 0}(\mathrm{RGC})=14.13$ and $\left(V-I_{\mathrm{c}}\right)_{0}(\mathrm{RGC})=1.04$, so the source has $I_{\mathrm{c} 0}(\mathrm{MOA} 192)=$ $19.84 \pm 0.24$ and $\left(V-I_{\mathrm{c}}\right)_{0}($ MOA 192$)=1.11 \pm 0.24($ see Bennett et al. 2008, for a detailed discussion of these values). Applying the appropriate $\left(I_{\mathrm{c}}, V-I_{\mathrm{c}}\right) \mathrm{SBC}$ relation from Table 3 gives an estimated angular diameter of $\theta_{\mathrm{LD}}(\mathrm{MOA} 192)=0.99 \pm 0.14 \mu \mathrm{as}$, which is resolved by the caustic crossing (or cusp approach).

\subsection{Comparison with other calibrations}

In their early publication, Barnes et al. (1978) obtained a linear SBC relation $F_{V}=3.964-0.333(B-V)$ for $0.10 \leqslant$ $(B-V) \leqslant 1.35$, with a typical residual scatter of $\sigma\left(F_{V}\right)= \pm 0.025$. Following K04, it can be expressed as $\log \left(\theta_{\mathrm{LD}}\right)=0.5134+$ $0.666(B-V)-0.2 V$. with a scatter of $\sigma\left(\log \theta_{\mathrm{LD}}\right)= \pm 0.05$, equivalent to $\sigma\left(\theta_{\mathrm{LD}}\right)= \pm 12 \%$. Over their common range of applicability, the relative difference $\rho$ in $\theta_{\mathrm{LD}}$ with our polynomial relation (coefficients listed in Table 3 ) is

$\rho(\mathrm{B} 78)=\left[\theta_{\mathrm{LD}}(\mathrm{B} 78)-\theta_{\mathrm{LD}}(\mathrm{K} 08)\right] / \theta_{\mathrm{LD}}(\mathrm{K} 08)=3.6 \pm 3.5 \%$.

We can also compare our ( $B, B-I)$ predictions (as an example) with the $(V, V-K)$ relations calibrated recently by Beuermann (2006, hereafter B06). The $S_{V}(V-K)$ relation of this author's Table 2 can be reformulated as:

$$
\log \theta_{\mathrm{LD}}=0.524(V-K)^{2}+0.267(V-K)-1.22110^{-3}-0.2 V \text {. }
$$

For the 34 stars in our sample, we obtain an average value of the relative difference with our calibration of $\rho(\mathrm{B} 06)=4.1 \pm 5.2 \%$. One should note that B06's calibration relies on several data sources, including indirect angular diameter estimates. The same comparison with K04's $(V, V-K)$ relation gives $\rho(\mathrm{K} 04)=0.7 \pm$ $4.6 \%$. The present calibration of the visible SBC relations is therefore compatible with previously determined SBC relations using visible and visible-IR color indices.

\section{Conclusion}

We computed polynomial SBC relations in $B V R_{\mathrm{c}} I_{\mathrm{c}}$ that can be used to predict the angular size of individual stars for which only broadband photometry is available. The visible-infrared linear relations established by $\mathrm{K} 04$ usually provide more accurate angular size estimates. However, in many cases the JHK band magnitudes of field stars are not available (due to crowding in 2MASS for instance), and the present $B V R_{\mathrm{c}} I_{\mathrm{c}}$ relations will provide a reliable photospheric angular diameter prediction with a typical uncertainty of $\approx 5 \%$. Their compatibility with visibleIR relations gives further confidence in their accuracy.

Acknowledgements. This research made use of the SIMBAD and VIZIER databases at the CDS (Strasbourg, France), the Hipparcos catalogues, the Lausanne General Catalogue of Photometric Data, the General Catalogue of Variable Stars, and NASA's ADS Bibliographic Services. We also received the support of PHASE, the high angular resolution partnership between ONERA, Observatoire de Paris, CNRS, and University Denis Diderot Paris 7.

\section{References}

Aufdenberg, J. P., Mérand, A., Coudé du Foresto, V., et al. 2006, ApJ, 645, 664, Erratum ApJ, 651, 677

Baines, E. K., McAlister, H. A., ten Brummelaar, T. A., et al. 2008, ApJ, 680, 728

Barnes, T. G., Evans, D. S., \& Moffett, T. J. 1978, MNRAS, 183, 285

Bennett, D. P., Bond, I. A., Udalski, A., et al. 2008, ApJ, 684, 663

Berger, D., Gies, D. R., McAlister, H. A., et al. 2006, ApJ, 644, 475

Bessell, M. S. 1990, A\&AS, 83, 357

Beuermann, K. 2006, A\&A, 460, 783

Bigot, L., Kervella, P., Thévenin, F., \& Ségransan, D. 2006, A\&A, 446, 635

Boden, A. F., Van Belle, G. T., \& Colavita, M. M. 1998, ApJ, 504, 39

Boyajian, T. S., McAlister, H. A., Baines, E. K., et al. 2008, ApJ, 683, 424

Celis, S. L. 1986, ApJS, 60, 879

Claret, A. 1998, A\&A, 335, 647

Claret, A. 2000, A\&A, 363, 1081

Claret, A. 2008, A\&A, 482, 259

Claret, A., Diaz-Cordoves, J., \& Gimenez, A. 1995, A\&AS, 114, 247

Cousins, A. W. J. 1980a, SAAO Circ., 1, 166

Cousins, A. W. J. 1980b, SAAO Circ., 1, 234

Davis, J., \& Tango, W. J. 1986, Nature, 323, 234

Dean, J. F., Warren, P. R., \& Cousins, A. W. J. 1978, MNRAS, 183, 569

Di Folco, E., Thévenin, F., Kervella, P., et al. 2004, A\&A, 426, 601

Ducati, J. R. 2002, NASA Ref. Pub., 1294

Fouqué, P., \& Gieren, W. P. 1997, A\&A, 320, 799

Groenewegen, M. A. T. 2004, MNRAS, 353, 903

Hanbury Brown, R., Davis, J., \& Allen, L. R. 1974, MNRAS, 167, 121

The Hipparcos Input Catalogue Version 2 1993, ESA SP-1136

The Hipparcos and Tycho Catalogues 1997, ESA SP-1200

Holmberg, J., Flynn, C., \& Portinari, L. 2006, MNRAS, 367, 449

Kervella, P., \& Domiciano de Souza, A. 2006, A\&A, 453, 1059

Kervella, P., Thévenin, F., Morel, P., Bordé, P., \& Di Folco, E. 2003a, A\&A, 408, 681

Kervella, P., Thévenin, F., Ségransan, D., et al. 2003b, A\&A, 404, 1087

Kervella, P., Thévenin, F., Morel, P., et al. 2004a, A\&A, 413, 251

Kervella, P., Thévenin, F., Di Folco, E., \& Ségransan, D. 2004b, A\&A, 426, 297, K04

Kervella, P., Bersier, D., Mourard, D., et al. 2004c, A\&A, 428, 587

Kervella, P., Mérand, A., Thévenin, F., et al. 2008, A\&A, submitted

Laing, J. D. 1989, SAAO Circ., 13, 29

Lane, B. F., Boden, A. F., \& Kulkarni, S. R. 2001, ApJ, 551, L81

Mermilliod, J.-C., Mermilliod, M., \& Hauck, B. 1997, A\&AS, 124, 349

http: //obswww . unige.ch/gcpd/gcpd.html

Monnier, J. D., Zhao, M., Pedretti, E., et al. 2007, Science, 317, 342

Mozurkewich, D., Armstrong, J. T., Hindsley, R. B., et al. 2003, AJ, 126, 2502

Nordgren, T. E., Germain, M. E., Benson, J. A., et al. 1999, AJ, 118, 3032

Nordgren, T. E., Sudol, J. J., \& Mozurkewich, D. 2001, AJ, 122, 2707

Nordgren, T. E., Lane, B. F., Hindsley, R. B., \& Kervella, P. 2002, AJ, 123, 3380

Peterson, D. M., Hummel, C. A., Pauls, T. A., et al. 2006, Nature, 440, 896

Pont, F., Gilliland, R. L., Moutou, C., et al. 2007, A\&A, 476, 1347

Samus, N. N. 2008, http: //www.sai.msu.su/groups/cluster/gcvs/

Ségransan, D., Kervella, P., Forveille, T., \& Queloz, D. 2003, A\&A, 397, L5

Skrutskie, R. M., Cutri, R., Stiening, M. D., et al. 2006, AJ, 131, 1163

Taylor, B. J. 2003, A\&A, 398, 721

ten Brummelaar, T. A., Mc Alister, H. A., Ridgway, S. T., et al. 2005, ApJ, 628, 453

Thé, P. S., Steenman, H. C., \& Alcaino, G. 1984, A\&A, 132, 385

Thévenin, F., Kervella, P., Pichon, B., et al. 2005, A\&A, 436, 253

Torres, G., Winn, J. N., \& Holman, M. J. 2008, ApJ, 677, 1324

van Belle, G. T. 1999, PASP, 111, 1515

van Belle, G. T., Lane, B. F., Thompson, R. R., et al. 1999, AJ, 117, 521

Yoo, J., DePoy, D. L., Gal-Yam, A., et al. 2004, ApJ, 603, 139 
P. Kervella and P. Fouqué: The angular sizes of dwarf stars and subgiants from $B V R_{\mathrm{c}} I_{\mathrm{c}}$ colors $(R N)$, Online Material $p 1$

Table 1. Interferometric data. The error bars are listed in small characters as exponents.

\begin{tabular}{|c|c|c|c|c|c|}
\hline Star & Spect. & $\lambda$ & $\theta_{\mathrm{UD}}$ (mas) & $\theta_{\mathrm{LD}}$ (mas) & Ref. \\
\hline$\alpha \mathrm{CMa} \mathrm{A}$ & A1V & B & $5.600^{0.070}$ & $5.896^{0.074}$ & 1 \\
\hline$\alpha \mathrm{CMa} \mathrm{A}$ & A1V & B & $5.630^{0.080}$ & $5.928^{0.084}$ & 2 \\
\hline$\alpha \mathrm{CMa} \mathrm{A}$ & A1V & K & $5.936^{0.016}$ & $6.039^{0.019}$ & 3 \\
\hline$\alpha \mathrm{CMa} \mathrm{A}$ & A1V & V & & $5.993^{0.108}$ & 4 \\
\hline$\alpha$ PsA & A3V & K & $2.197^{0.023}$ & $2.228^{0.023}$ & 5 \\
\hline$\beta$ Leo & A3V & K & $1.429^{0.027}$ & $1.449^{0.027}$ & 5 \\
\hline 94 Cet & F8V & K & $0.774^{0.026}$ & $0.788^{0.026}$ & 6 \\
\hline$\alpha$ CMi A & F5IV-V & V & $5.190^{0.040}$ & $5.430^{0.070}$ & 7 \\
\hline$\alpha$ CMi A & F5IV-V & V & & $5.446^{0.054}$ & 4 \\
\hline$\alpha$ CMi A & F5IV-V & K & $5.376^{0.047}$ & $5.448^{0.053}$ & 8 \\
\hline$\tau$ Boo & F7V & K & $0.771^{0.015}$ & $0.786^{0.016}$ & 6 \\
\hline$v$ And & F8V & K & $1.091^{0.009}$ & $1.114^{0.009}$ & 6 \\
\hline$\eta$ Boo & G0IV & V & $2.170^{0.060}$ & $2.280^{0.070}$ & 7 \\
\hline$\eta$ Boo & G0IV & V & & $2.269^{0.025}$ & 4 \\
\hline$\eta$ Boo & G0IV & K & & $2.200^{0.031}$ & 9 \\
\hline$\mu \mathrm{Her}$ & G5IV & V & & $1.953^{0.039}$ & 4 \\
\hline$\zeta$ Her A & G0IV & V & & $2.367^{0.051}$ & 4 \\
\hline$\zeta$ Her A & G0IV & V & $2.370^{0.080}$ & $2.490^{0.090}$ & 7 \\
\hline$\alpha$ Cen A & $\mathrm{G} 2 \mathrm{~V}$ & K & $8.314^{0.016}$ & $8.511^{0.020}$ & 10 \\
\hline Sun & G2V & & & $1919260^{10}$ & \\
\hline 70 Vir & G5V & K & $0.986^{0.023}$ & $1.009^{0.024}$ & 6 \\
\hline$\mu$ Cas A & G5Vp & K & $0.951^{0.009}$ & $0.973^{0.009}$ & 11 \\
\hline HR 7670 & G6IV & K & $0.682^{0.019}$ & $0.698^{0.019}$ & 6 \\
\hline $55 \mathrm{Cnc}$ & G8V & K & $0.834^{0.024}$ & $0.854^{0.024}$ & 6 \\
\hline$\beta \mathrm{Aql}$ & G8IV & V & $2.070^{0.090}$ & $2.180^{0.090}$ & 12 \\
\hline$\tau$ Cet & G8V & K & $2.032^{0.031}$ & $2.078^{0.031}$ & 5 \\
\hline 54 Psc & K0V & K & $0.773^{0.026}$ & $0.790^{0.027}$ & 6 \\
\hline$\sigma$ Dra & KOV & K & $1.224^{0.011}$ & $1.254^{0.012}$ & 11 \\
\hline HR 511 & K0V & K & $0.747^{0.021}$ & $0.763^{0.021}$ & 11 \\
\hline$\delta$ Eri & KOIV & K & & $2.394^{0.029}$ & 9 \\
\hline$\eta$ Cep & KOIV & V & $2.510^{0.040}$ & $2.650^{0.040}$ & 12 \\
\hline$\alpha$ Cen B & K1V & K & $5.881^{0.021}$ & $6.000^{0.021}$ & 13 \\
\hline GJ 166A & K1V & K & $1.600^{0.060}$ & $1.650^{0.060}$ & 14 \\
\hline$\epsilon$ Eri & $\mathrm{K} 2 \mathrm{~V}$ & K & $2.093^{0.029}$ & $2.148^{0.029}$ & 5 \\
\hline GJ 570A & K4V & K & $1.190^{0.030}$ & $1.230^{0.030}$ & 14 \\
\hline GJ 845 & $\mathrm{~K} 4.5 \mathrm{~V}$ & K & $1.840^{0.020}$ & $1.890^{0.020}$ & 14 \\
\hline 61 Cyg A & K5V & K & & $1.775^{0.013}$ & 15 \\
\hline 61 Cyg B & K7V & K & & $1.581^{0.022}$ & 15 \\
\hline GJ 380 & K7V & HK & $1.268^{0.040}$ & $1.175^{0.040}$ & 16 \\
\hline GJ 887 & $\mathrm{M} 0.5 \mathrm{~V}$ & K & $1.366^{0.040}$ & $1.388^{0.040}$ & 17 \\
\hline GJ 411 & M1.5V & HK & $1.413^{0.030}$ & $1.464^{0.030}$ & 16 \\
\hline GJ 699 & M4Ve & HK & $0.987^{0.040}$ & $1.026^{0.040}$ & 16 \\
\hline
\end{tabular}

References: (1) Hanbury Brown et al. 1974; (2) Davis \& Tango 1986. (3) Kervella et al. 2003a; (4) Mozurkewich et al. 2003; (5) Di Folco et al. 2004; (6) Baines et al. 2008; (7) Nordgren et al. 2001; (8) Kervella et al. 2004a; (9) Thévenin et al. 2005; (10) Kervella et al. 2003b; (11) Boyajian et al. 2008. (12) Nordgren et al. 1999; (13) Bigot et al. 2006; (14) Kervella et al. 2004b; (15) Kervella et al. 2008; (16) Lane et al. 2001; (17) Ségransan et al. 2003.
Table 2. Photometric data. When the uncertainty on the photometric measurements was not available, we adopted an arbitrary \pm 0.05 value.

\begin{tabular}{|c|c|c|c|c|c|}
\hline Star & $B$ & $V$ & $R_{\mathrm{c}}$ & $I_{\mathrm{c}}$ & Ref. \\
\hline$\alpha \mathrm{CMaA}$ & $-1.43^{0.01}$ & $-1.44^{0.01}$ & $-1.43^{0.05}$ & $-1.42^{0.05}$ & $a b$ \\
\hline$\alpha \mathrm{PsA}$ & $1.25^{0.02}$ & $1.17^{0.02}$ & $1.11^{0.05}$ & $1.09^{0.05}$ & $\mathrm{bc}$ \\
\hline$\beta$ Leo & $2.23^{0.01}$ & $2.14^{0.01}$ & & $2.04^{0.05}$ & $\mathrm{H}$ \\
\hline $94 \mathrm{Cet}$ & $5.65^{0.01}$ & $5.07^{0.01}$ & $4.75^{0.05}$ & $4.44^{0.05}$ & $\mathrm{c}$ \\
\hline$\alpha \mathrm{CMi} \mathrm{A}$ & $0.83^{0.03}$ & $0.40^{0.03}$ & $0.15^{0.06}$ & $-0.09^{0.06}$ & $b c$ \\
\hline$\tau$ Boo & $5.00^{0.03}$ & $4.49^{0.03}$ & & $3.98^{0.04}$ & $\mathrm{C}$ \\
\hline$v$ And & $4.63^{0.01}$ & $4.09^{0.01}$ & & $3.51^{0.03}$ & $\mathrm{C}$ \\
\hline$\eta$ Boo & $3.26^{0.01}$ & $2.68^{0.01}$ & & $2.03^{0.05}$ & $\mathrm{H}$ \\
\hline$\mu \mathrm{Her}$ & $4.16^{0.03}$ & $3.41^{0.03}$ & & $2.70^{0.04}$ & G \\
\hline$\zeta$ Her A & $3.46^{0.01}$ & $2.81^{0.01}$ & & $2.11^{0.03}$ & $\mathrm{G}$ \\
\hline$\alpha$ Cen A & $0.70^{0.05}$ & $-0.01^{0.03}$ & $-0.37^{0.06}$ & $-0.70^{0.06}$ & $\mathrm{a}$ \\
\hline Sun & $-26.11^{0.02}$ & $-26.75^{0.01}$ & $-27.10^{0.01}$ & $-27.44^{0.01}$ & S \\
\hline 70 Vir & $5.69^{0.01}$ & $4.98^{0.01}$ & & $4.21^{0.05}$ & G \\
\hline$\mu \mathrm{Cas} \mathrm{A}$ & $5.87^{0.03}$ & $5.17^{0.03}$ & & $4.34^{0.04}$ & G \\
\hline HR 7670 & $6.50^{0.01}$ & $5.75^{0.01}$ & & $4.92^{0.07}$ & $\mathrm{~F}$ \\
\hline $55 \mathrm{Cnc}$ & $6.83^{0.02}$ & $5.96^{0.01}$ & & $5.13^{0.07}$ & $\mathrm{~F}^{*}$ \\
\hline$\beta \mathrm{Aql}$ & $4.57^{0.01}$ & $3.72^{0.01}$ & $3.26^{0.05}$ & $2.83^{0.05}$ & $\mathrm{bc}$ \\
\hline$\tau$ Cet & $4.22^{0.01}$ & $3.50^{0.01}$ & $3.07^{0.05}$ & $2.68^{0.05}$ & bc \\
\hline 54 Psc & $6.73^{0.02}$ & $5.88^{0.02}$ & & $5.05^{0.02}$ & G \\
\hline$\sigma$ Dra & $5.46^{0.01}$ & $4.68^{0.01}$ & & $3.83^{0.02}$ & $\mathrm{G}$ \\
\hline HR 511 & $6.43^{0.01}$ & $5.63^{0.01}$ & & $4.82^{0.05}$ & $\mathrm{G}$ \\
\hline$\delta$ Eri & $4.44^{0.02}$ & $3.53^{0.01}$ & $3.02^{0.05}$ & $2.59^{0.05}$ & bc \\
\hline$\eta$ Cep & $4.34^{0.01}$ & $3.43^{0.01}$ & & $2.49^{0.04}$ & $\mathrm{G}$ \\
\hline$\alpha$ Cen B & $2.25^{0.04}$ & $1.35^{0.03}$ & $0.88^{0.06}$ & $0.47^{0.06}$ & $\mathrm{a}$ \\
\hline GJ 166A & $5.25^{0.01}$ & $4.43^{0.01}$ & $3.96^{0.05}$ & $3.54^{0.05}$ & bc \\
\hline$\epsilon$ Eri & $4.61^{0.01}$ & $3.73^{0.01}$ & $3.22^{0.05}$ & $2.78^{0.05}$ & $\mathrm{bc}$ \\
\hline GJ 570A & $6.75^{0.03}$ & $5.72^{0.03}$ & $5.07^{0.06}$ & $4.54^{0.06}$ & $\mathrm{~d}$ \\
\hline GJ 845 & $5.74^{0.02}$ & $4.69^{0.01}$ & $4.06^{0.05}$ & $3.54^{0.05}$ & bcd \\
\hline 61 Cyg A & $6.27^{0.03}$ & $5.20^{0.03}$ & & $3.89^{0.04}$ & $\mathrm{C}^{*}$ \\
\hline 61 Cyg B & $7.41^{0.03}$ & $6.06^{0.02}$ & & $4.50^{0.04}$ & $\mathrm{C}^{*}$ \\
\hline GJ 380 & $7.97^{0.02}$ & $6.60^{0.01}$ & & $5.09^{0.02}$ & $\mathrm{I}^{*}$ \\
\hline GJ 887 & $8.83^{0.01}$ & $7.35^{0.01}$ & $6.38^{0.05}$ & $5.33^{0.05}$ & ce \\
\hline GJ 411 & $9.00^{0.02}$ & $7.50^{0.01}$ & $6.49^{0.05}$ & $5.35^{0.05}$ & $\mathrm{~d}$ \\
\hline GJ 699 & $11.11^{0.03}$ & $9.54^{0.03}$ & $8.31^{0.06}$ & $6.76^{0.06}$ & acdf \\
\hline
\end{tabular}

References: (a) Bessell 1990; (b) Cousins 1980b; (c) Cousins 1980a; (d) Celis 1986; (e) Thé 1984; (f) Laing 1989; (S) Holmberg et al. 2006; (C) Hipparcos catalogue, Field H42 = C; (C*) for 61 Cyg A and B, we transformed $(V-I)_{j}$ from Ducati $(2002)$ to $(V-I)_{\mathrm{c}}$ according to Hipparcos precepts when Field H42 = C; (F) Hipparcos catalogue, Field $\mathrm{H} 42=\mathrm{F} ;\left(\mathrm{F}^{*}\right)$ for $55 \mathrm{Cnc}$, we converted $(R-I)_{\mathrm{c}}=0.388 \pm 0.003$ from Taylor (2003) to $(V-I)_{\mathrm{c}}$ according to Hipparcos precepts when Field H42 = F; (G) Hipparcos catalogue, Field H42 = G; (H) Hipparcos catalogue, Field H42 $=\mathrm{H}$; (I*) for GJ380, we converted $(B-V)_{t}$ from Tycho to $(V-I)_{\mathrm{c}}$ according to Hipparcos precepts when Field $\mathrm{H} 42=\mathrm{I}$. 